\title{
Impact of sphingomyelin acyl chain (16:0 vs 24:1) on the interfacial properties of Langmuir monolayers: A PM-IRRAS study
}

\author{
Romina F. Vázquez ${ }^{\mathrm{a}, \mathrm{b}}$, M. Antonieta Daza Millone ${ }^{\mathrm{c}}$, Felippe J. Pavinatto ${ }^{\mathrm{d}, 1}$, María L. Fanani ${ }^{\mathrm{e}, \mathrm{f}}$, \\ Osvaldo N. Oliveira Jr. ${ }^{\mathrm{d}}$, María E. Vela ${ }^{\mathrm{c}}$, Sabina M. Maté ${ }^{\mathrm{a}, *}$ \\ a Instituto de Investigaciones Bioquímicas de La Plata (INIBIOLP), CCT- La Plata, CONICET, Facultad de Ciencias Médicas, Universidad Nacional de La Plata, 60 y 120, \\ (1900), La Plata, Argentina \\ b Departamento de Química, Facultad de Ciencias Exactas, Universidad Nacional de La Plata, 47 y 115, (1900), La Plata, Argentina \\ ${ }^{\mathrm{c}}$ Instituto de Investigaciones Fisicoquímicas Teóricas y Aplicadas (INIFTA), CCT- La Plata, CONICET, Universidad Nacional de La Plata, Sucursal 4 Casilla de Correo 16, \\ (1900), La Plata, Argentina \\ ${ }^{\mathrm{d}}$ São Carlos Institute of Physics, University of São Paulo, SP, Brazil \\ e Centro de Investigaciones en Química Biológica de Córdoba (CIQUIBIC), CONICET, Argentina \\ ${ }^{\mathrm{f}}$ Departamento de Química Biológica Ranwel Caputto, Facultad de Cs. Químicas, Universidad Nacional de Córdoba. Haya de la Torre y Medina Allende, Ciudad \\ Universitaria, Córdoba, Argentina
}

\section{A R T I C L E I N F O}

\section{Keywords:}

Membrane rafts

Cholesterol-sphingomyelin interactions

Langmuir monolayers

PM-IRRAS

\begin{abstract}
A B S T R A C T
Membrane structure is a key factor for the cell`s physiology, pathology, and therapy. Evaluating the importance of lipid species such as N-nervonoyl sphingomyelin (24:1-SM) —able to prevent phase separation- to membrane structuring remains a formidable challenge. This is the first report in which polarization-modulated infrared reflection-absorption spectroscopy (PM-IRRAS) is applied to investigate the lipid-lipid interactions in 16:0 vs 24:1-SM monolayers and their mixtures with 1,2-Dioleoyl-sn-glycero-3-phosphocholine (DOPC) and cholesterol (Chol) (DOPC/SM/Chol 2:1:1). From the results we inferred that the cis double bond $(\Delta 15)$ in 24:1-SM molecule diminishes intermolecular H-bonding and chain packing density compared to that of 16:0-SM. In ternary mixtures containing 16:0-SM, the relative intensity of the two components of the Amide I band reflected changes in the H-bonding network due to SM-Chol interactions. In contrast, the contribution of the main components of the Amide I band in DOPC/24:1-SM/Chol remained as in 24:1-SM monolayers, with a larger contribution of the non-H-bonded component. The most interesting feature in these ternary films is that the $\mathrm{C}=$ O stretching mode of DOPC appeared with an intensity similar to that of SM Amide I band in DOPC/16:0-SM/ Chol monolayers (a two-phase $[\mathrm{Lo} / \mathrm{Le}]$ system), whereas an extremely low intensity of the $\mathrm{C}=\mathrm{O}$ band was detected in DOPC/24:1-SM/Chol monolayers (single Le phase). This is evidence that the unsaturation in 24:1-SM affected not only the conformational properties of acyl chains but also the orientation of the chemical groups at the air/water interface. The physical properties and overall H-bonding ability conferred by 24:1-SM may have implications in cell signaling and binding of biomolecules.
\end{abstract}

\section{Introduction}

Cellular membranes are highly organized structures that separate the intracellular from the extracellular environment and create discrete compartments within living cells. The structures and functions of these membranes are primarily based on the intrinsic properties of membrane lipids. Current views on structural and dynamical aspects of biological membranes have challenged the idea of lipids forming a randomly organized fluid bilayer matrix [1]. The concept of an organization of the lipid components of membranes into domains has been introduced which constitutes the basis of the lipid raft hypothesis [2]. Sphingomyelin (SM) is one of the key constituents of a lipid raft [3], which is a segregated domain in the cell membrane where SMs, glycosphingolipids, cholesterol (Chol) and specific proteins are selectively assembled. Lipid rafts have been implicated in crucial cellular functions as membrane trafficking, cell signaling, and binding of biomolecules [4]. SM and related sphingolipids are believed to have important functional role(s) on membrane domain formation, e.g. for the Chol-rich

\footnotetext{
* Corresponding author.

E-mail address: smate@med.unlp.edu.ar (S.M. Maté).

${ }^{1}$ Current permanent address: Clean Energy Institute, University of Washington - WA, Seattle, USA.
} 
liquid-ordered (Lo) phase [5,6]. This phase is an intermediate physical state with tight lipid packing while maintaining rotational and lateral lipid mobility, leading to a thicker, but still liquid crystalline membrane [7]. The interaction of Chol with SM and phosphatidylcholine (PC) has been studied in monolayers and bilayers as membrane models, in which the effects of Chol were found to depend on several factors, including the phospholipid headgroup, chain length, lipid backbone and the presence of saturated or unsaturated chains [8-10]. Chol and SM have been proposed to interact via hydrogen bonding involving the hydroxyl group of Chol and the amide group of the sphingosine, and through hydrophobic interactions between the rigid Chol rings and the SM acyl chains. As a consequence of these distinctive interactions, Lo phases enriched in SM and Chol coexist with liquid-disordered $(L d)$ phases in model membranes containing PC, SM, and Chol over a wide range of compositions [11].

Naturally occurring SMs have a phosphocholine headgroup esterified to the $\mathrm{C} 1$ hydroxyl group of a long-chain sphingoid base. The most common long chain base in mammalian SM is sphingosine. Typically, the acyl chains amide-linked to carbon 2 of the sphingoid base are long (16-24 carbon atoms) and saturated, but 24:1 has also been recognized as a common N-linked acyl chain in natural SMs [12]. 24:1-SM is the most common unsaturated SM species, accounting for $20-25 \%$ of SM in bovine brain and HL-60 cells [13,14]. Changing levels of 24:1-SM have been linked to processes in the central nervous system such as myelin maturation [15], pointing to the physiological importance of acyl compositional changes in SMs. The influence of 24:1-SM on the lateral organization and mechanical stability of model and natural membranes has been examined [16], where confocal and atomic force microscopy images showed that unlike 16:0-SM and 24:0-SM, 24:1-SM does not induce phase segregation in ternary lipid mixtures with dioleoyl-PC (DOPC) and Chol. It seems that 24:1-SM is able to accommodate both DOPC and Chol in a single phase. The 24:1-SM behavior reinforces the idea by Epand and Epand that the driving force behind creation of larger clusters or domains is not only SM-Chol interactions [17]. In fact, the exact nature of the molecular interactions that lead to lipid immiscibilities in membranes is a question partly unresolved. The distinct features in the molecular structure of SM may have substantial effects on its interactions with other membrane components such as sterols and glycerophospholipids. For example, the capacity of SM to form intraand intermolecular hydrogen bonds has an important role to play in the phase behavior of SMs [18,19].

In this study we further characterize SM-SM (24:1 vs 16:0) and SM/ PC/Chol monolayers to understand lipid interactions that drastically alter membrane structure and functions. Specifically, we performed PM-IRRAS measurements on lipid monolayers to characterize the conformational properties and intermolecular interactions in pure lipid films and in two ternary lipid mixtures (DOPC/16:0-SM/Chol and DOPC/24:1-SM/Chol)—which are relevant as mammalian cell membrane models. We analyze the importance of the acyl chain composition of SM in establishing SM-colipid interactions for the formation of membrane domains. PM-IRRAS and BAM results showed that the presence of the cis double bond in 24:1-SM molecule affects not only the conformational properties of acyl chains but also the orientation of the chemical groups at the air/water interface, weakening the intermolecular interactions between neighboring molecules and inducing a disordering effect on DOPC/24:1-SM/Chol ternary systems, preventing phase segregation. We advocate that investigating lipid interactions and phase behavior will bring us closer to understanding how domains and rafts are formed in biological membranes. The different properties of the two ternary systems assayed in this work could have significant implications for membrane proteins functions and binding of biomolecules.

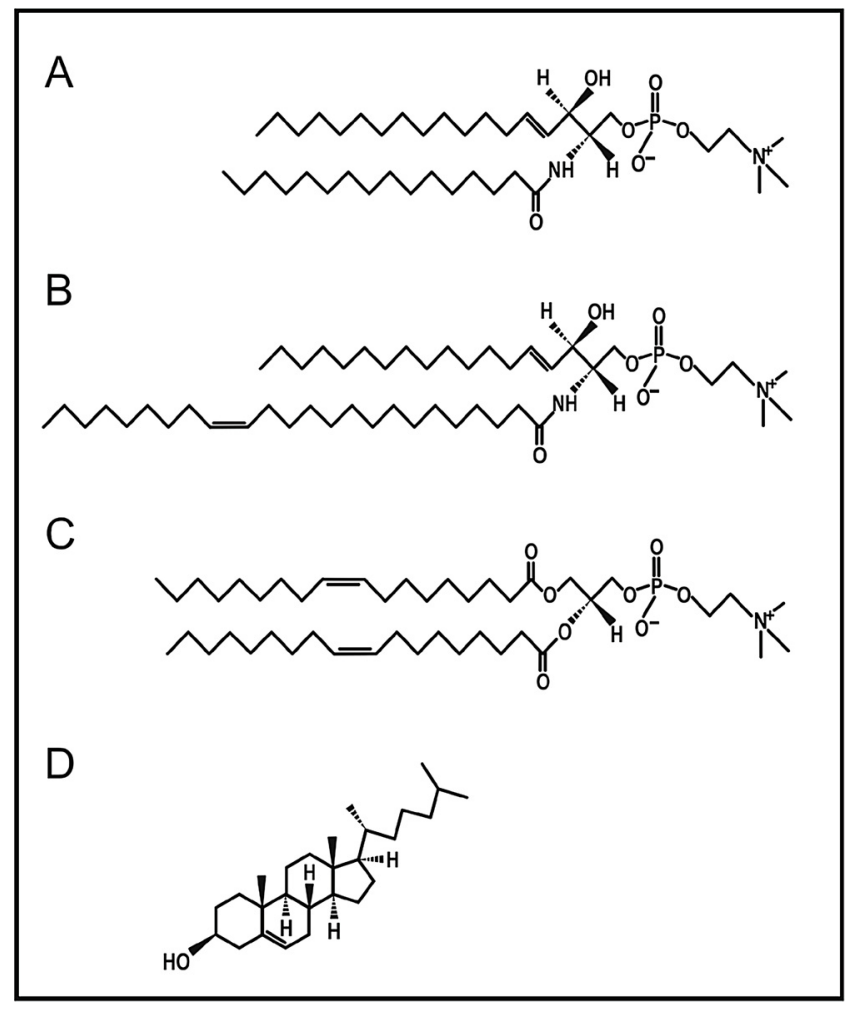

Fig. 1. Chemical structure of lipid molecules used. SMs with a chain length of $\mathrm{n}=16: 0$ (palmitoyl-SM) (A) and $\mathrm{n}=24: 1$ (nervonoyl-SM) (B), 1,2-dioleoyl-snglycero-3-phosphocholine (DOPC) (C) and Chol (D). The sphingosine backbone of SM has double-bonded carbons at the $4^{\text {th }}$ and $5^{\text {th }}$ positions, whilst the fatty acyl-chain is bonded to the backbone by an amide bond. The amide and hydroxyl groups of SM serve as both hydrogen bond donors and acceptors.

\section{Experimental section}

\subsection{Materials}

1,2-Dioleoyl-sn-glycero-3-phosphocholine (DOPC), N-palmitoyl-Derythro-sphingosyl phosphorylcholine (16:0-SM), N-nervonoyl-D-erythro-sphingosyl phosphorylcholine (24:1-SM), and cholesterol (Chol) were purchased from Avanti Polar Lipids (Birmingham, AL, USA) and used without further purification. The molecular structures of lipids used in this study are shown in Fig. 1 . Tris buffer, $\mathrm{NaCl}, \mathrm{CaCl}_{2}$ and other reagents, all analytical-grade, were acquired from Sigma-Aldrich (St. Louis, MO, USA), unless otherwise stated. HPLC-grade chloroform was purchased from Merck (Darmstadt, Germany). The surface tension and resistivity of the ultrapure water used were $72.2 \mathrm{mN} / \mathrm{m}$ and $18.2 \mathrm{M} \Omega$ $\mathrm{cm}$ at $23^{\circ} \mathrm{C}$, respectively.

\subsection{Surface-pressure $(\pi)$ experiments}

Surface pressure measurements were carried out with a Langmuir trough by NIMA Model 102 A (NIMA Technology, Coventry, UK) with a Wilhelmy platinum plate as the $\pi$ sensor. The aqueous subphase consisted of $20 \mathrm{mM}$ Tris, $150 \mathrm{mM} \mathrm{NaCl}, 2 \mathrm{mM} \mathrm{CaCl}_{2}$, pH 7.4 (TBS Buffer) prepared in ultrapure water. The lipid dissolved in chloroform was gently spread over the subphase surface with a Hamilton microsyringe. After $15 \mathrm{~min}$ of solvent evaporation and monolayer equilibration, the films were compressed with two symmetric moving barriers at a compression rate of $5 \mathrm{~cm}^{2}$. $\mathrm{min}^{-1}$. All the surface pressure measurements were performed at $23 \pm 1{ }^{\circ} \mathrm{C}$ and repeated at least three times to ensure reproducibility. The in-plane elasticity of the films was analyzed by calculating the compressibility modulus $\left(\mathrm{Cs}^{-1}\right)$ from the $\pi$-Area isotherms according to Eq. (1) [20]: 

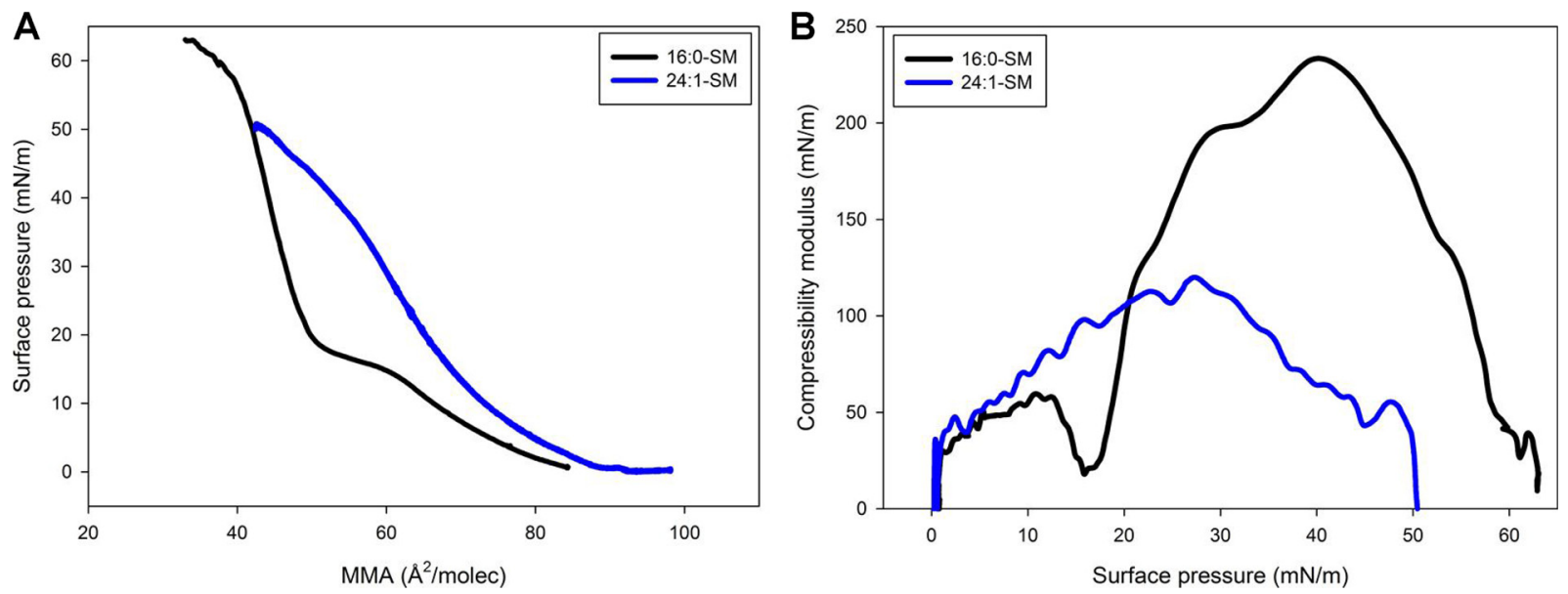

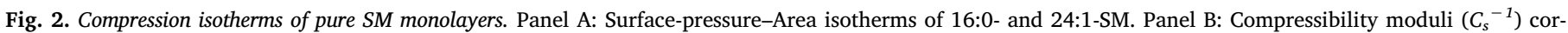
responding to each isotherm, calculated according to Eq. (1).

$C s^{-1}=-\mathrm{A}(\partial \pi / \partial \mathrm{A})$

where A is the mean molecular area (MMA) at the surface pressure $\pi$.

\subsection{Brewster angle microscopy (BAM)}

Langmuir monolayers were prepared using a KSV Minitrough equipment (KSV, Helsinski, Finland) mounted on the stage of the microscope. Film imaging by BAM was performed with an imaging ellipsometer (Nanofilm EP3sw imaging ellipsometer, Accurion GmbH, Gottingen, Germany) equipped with a $532 \mathrm{~nm}$ laser, a $20 \times$ objective, and a CCD camera operating at a resolution of $2 \mu \mathrm{m}$ in the BAM mode. Minimum reflection was set with a polarized laser beam $(\lambda=532 \mathrm{~nm})$ incident on the bare aqueous surface at the experimentally calibrated Brewster angle $\left(\sim 53.1^{\circ}\right)$. The lipid monolayers were spread onto the aqueous surface and compressed to the desired pressure. After monolayer formation and during compression, the reflected light was collected through a $20 \times$ objective to a CCD camera. The gray level at each pixel of the BAM images can be converted to reflectivity values with calibration factors tested for each individual experiment. The reflectivity obtained from BAM measurements is related to both the film thickness and refractive index of the film [21]. Therefore, the liquidexpanded ( $L e$ ) phases of lipid films appear as dark gray areas whilst the more compact and thicker Lo phase is observed as light gray domains.

\subsection{Polarization-modulated infrared reflection-absorption spectroscopy (PM-IRRAS) experiments}

PM-IRRAS measurements were performed using a KSV PMI 550 instrument (KSV, Biolin Scientific Oy, Espoo, Finland) with the experimental setup described by Pavinatto et al [22]. In the PM-IRRAS technique, the polarized reflectivities for the parallel (Rp) and perpendicular (Rs) directions to the plane of incidence are measured simultaneously by using an incoming light that is continuously modulated at a high frequency between $s$ - and $p$-polarization. The absorption of the $p$-polarized light comes mainly from vertically oriented dipoles, while that of the $s$-polarized light comes from the horizontally oriented ones. A differential reflectivity spectrum $\Delta R=(R p-R s) /(R p+R s)$ is then obtained which provides information on the orientation of the vibrational dipoles at the surface. PM-IRRAS measurements were performed at an incident angle of $80^{\circ}$, at which positive bands indicate a transition moment oriented preferentially on the surface plane, whereas negative bands indicate preferential orientation perpendicular to the surface. An average of 600 scans was collected for each spectrum at a resolution of $8 \mathrm{~cm}^{-1}$. The spectra of the monolayers were normalized to the respective subphase spectra. The lipid monolayers were obtained by spreading the pure lipids or the lipid mixtures (DOPC/16:0-SM/Chol or DOPC/24:1-SM/Chol, at 2:1:1 molar ratio) dissolved in chloroform on the subphase (TBS Buffer) surface. After solvent evaporation and monolayer equilibration, the monolayers were compressed at $5 \mathrm{~cm}^{2} \cdot \mathrm{min}^{-1}$ until the desired lateral pressure was achieved and PMIRRAS spectra were collected $15 \mathrm{~min}$ after the monolayer equilibration. Measurements were carried out in a class 10,000 clean room at $23 \pm 1{ }^{\circ} \mathrm{C}$. Representative PM-IRRAS spectra of two independent experiments are shown.

\section{Results and discussion}

The main aim in this paper is to investigate by means of PM-IRRAS measurements the influence of unsaturation in the hydrophobic chain of 24:1-SM for its monolayer properties, in addition to comparing the ternary mixtures involving DOPC, Chol and SMs. The results are therefore organized into two subsections, dealing with the neat 16:0-SM and 24:1-SM monolayers and those made with the ternary mixtures. In each subsection, we start the analysis with a full characterization of the monolayer compression isotherms, their stability and rheological properties, so as to link those results to the data obtained from the PMIRRAS measurements.

\subsection{Neat 16:0 and 24:1-SM systems}

The $\pi-\mathrm{A}$ isotherms on panel A in Fig. 2 for pure 16:0-SM and 24:1SM monolayers are similar to those reported in the literature with collapse at $62.1 \pm 2.0 \mathrm{mN} / \mathrm{m}$ and $50.0 \pm 0.6 \mathrm{mN} / \mathrm{m}$, respectively [23,24]. The larger MMA values of 24:1-SM along the whole isotherm reflect a lateral packing density significantly lower than for 16:0-SM. Pure 16:0-SM monolayers exhibit a plateau at $15 \mathrm{mN} / \mathrm{m}$ typical of a phase transition, a feature that was not observed for 24:1-SM. The compressibility modulus $\left(C_{s}^{-1}\right)$ provides insights into the lateral packing elasticity, i.e., ease/resistance to lateral compression, within the monolayer. Typical $C_{s}^{-1}$ values for Le phases are $\sim 100 \mathrm{mN} / \mathrm{m}$ or lower, while higher values denote liquid condensed $(L c$ ) (and $L o$ ) phases. The surface elasticity data on panel B in Fig. 2 indicate a transition between $L e$ and $L c$ phases at $\sim 15 \mathrm{mN} / \mathrm{m}$ for 16:0-SM films, whereas 24:1-SM monolayers formed $L e$ films with no indication of $2 \mathrm{D}$ phase transitions. At high surface pressures, such as those that mimic membrane-like conditions [25], $C_{s}^{-1}$ values for 24:1-SM were consistently lower than those of 16:0-SM. At a surface pressure of $30 \mathrm{mN} /$ $\mathrm{m}, C_{s}^{-1}=84 \pm 18 \mathrm{mN} / \mathrm{m}$ indicated a $L e$ phase state for $24: 1-\mathrm{SM}$ monolayer while a $C_{s}^{-1}=203 \pm 22 \mathrm{mN} / \mathrm{m}$ for 16:0-SM corresponds to a $L c$ phase. These results indicate that changes in the acyl 
A

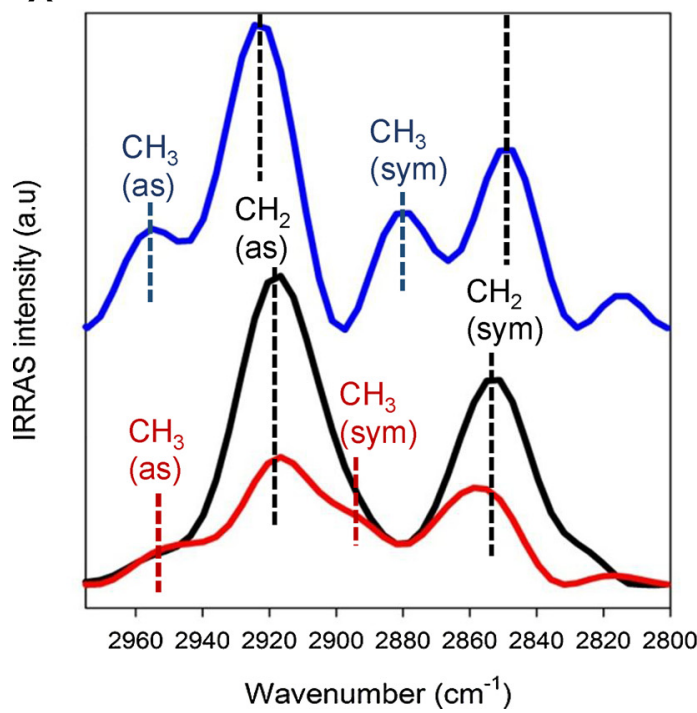

B

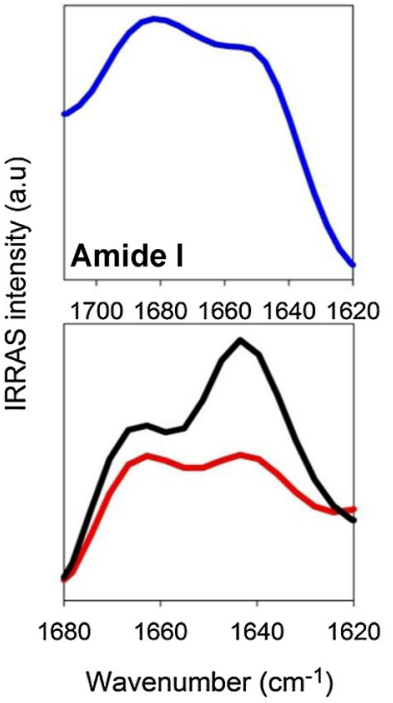

C

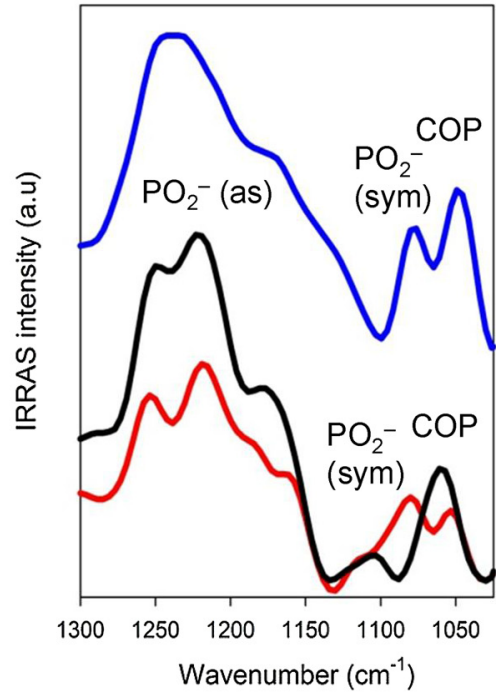

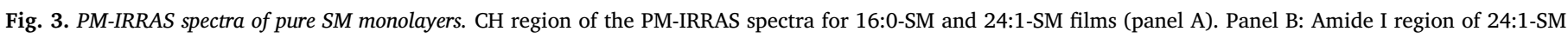

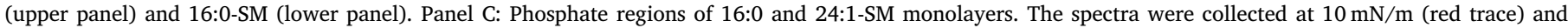

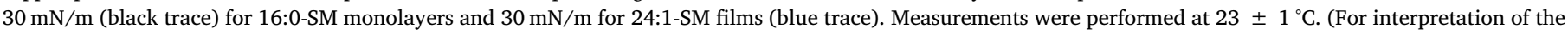
references to colour in this figure legend, the reader is referred to the web version of this article).

composition of SMs markedly affect their monolayer in-plane elasticity.

The differences between pure 16:0 and 24:1-SM monolayers are likely to be a consequence of a closer lipid packing and higher $\mathrm{H}$ bonding ability with neighboring SMs for 16:0-SM in comparison to 24:1-SM. We performed PM-IRRAS measurements to characterize the conformational properties of these SMs, and Fig. 3 shows the spectra taken at $30 \mathrm{mN} / \mathrm{m}$. For $16: 0-\mathrm{SM}$ the spectrum at $10 \mathrm{mN} / \mathrm{m}$ was also obtained to analyze the differences in the IR absorption characteristics of 16:0-SM monolayers in the $L c$ (chain-ordered) and $L e$ (chain-disordered) phases. In the CH-region of the PM-IRRAS spectra of pure 16:0-SM monolayers in the $L e$ phase $(10 \mathrm{mN} / \mathrm{m})$ two main bands appeared at 2916 and $2858 \mathrm{~cm}^{-1}$ (Fig. 3, panel A) assigned to $\mathrm{CH}_{2}$ antisymmetric and symmetric stretching modes, respectively. Bands assigned to antisymmetric and symmetric stretching of $\mathrm{CH}_{3}$ also appeared at 2953 and $2887 \mathrm{~cm}^{-1}$. Upon compression to $30 \mathrm{mN} / \mathrm{m}$, the $16: 0-\mathrm{SM}$ films ( $L c$ phase) displayed two well-defined, intense peaks at 2916 and $2851 \mathrm{~cm}^{-1}$ corresponding to $\mathrm{CH}_{2}$ antisymmetric and symmetric stretching modes, respectively, and a small shoulder at $2953 \mathrm{~cm}^{-1}$ is due to $\mathrm{CH}_{3}$ stretching. The large increase in the $\mathrm{CH}_{2}$-to- $\mathrm{CH}_{3}$ intensity ratio correlates with an increased order in the acyl chains when 16:0SM monolayers underwent the $L e$-to- $L c$ phase transition. In contrast, the spectra of pure 24:1-SM monolayers at $30 \mathrm{mN} / \mathrm{m}$ show $\mathrm{CH}_{2}$ bands at 2920 (antisymmetric stretching) and $2854 \mathrm{~cm}^{-1}$ (symmetric stretching) and intense bands at 2954 and $2878 \mathrm{~cm}^{-1}$ owing to $\mathrm{CH}_{3}$ antisymmetric and symmetric stretching modes, respectively. As expected, 16:0-SM formed highly ordered lipid monolayers at $30 \mathrm{mN} / \mathrm{m}$ while the higher frequency of $\mathrm{CH}_{2}$ bands and the presence of $\mathrm{CH}_{3}$ bands in 24:1-SM films spectra are characteristic of conformational disorder in the acyl chains [26-28].

In the Amide I region of 16:0-SM films (Fig. 3B, lower panel), two bands were observed at 1662 and $1643 \mathrm{~cm}^{-1}$ at $10 \mathrm{mN} / \mathrm{m}$ (Le phase), which could be assigned to non- or weakly-H-bonded and H-bonded amide groups, respectively [29-31]. For 16:0-SM monolayers in the $L c$ phase, the two bands remained but with an increment in intensity of the $1643 \mathrm{~cm}^{-1}$ band, thus indicating a higher level of intermolecular $\mathrm{H}$ bonded lipids at $30 \mathrm{mN} / \mathrm{m}$. Deconvolution of the Amide I band of 16:0SM indicated a $50 \%$ of $\mathrm{H}$-bonded groups at $10 \mathrm{mN} / \mathrm{m}$, whereas this component increased to $66 \%$ when monolayers were compressed to $30 \mathrm{mN} / \mathrm{m}$ (Figs. S1A and S1B in the Supplementary Material). This result is in line with the intensity increase of the high wavenumber component with temperature, along the transition from the gel to the $L d$ phase reported for egg sphingomyelin liposomes [30]. As for 24:1-SM films at $30 \mathrm{mN} / \mathrm{m}$ (upper panel in Fig. 3B), the asymmetric Amide I band had two components at 1682 and $1650 \mathrm{~cm}^{-1}$, with the high wavenumber component associated with non-H-bonded SM being more intense than that of the $\mathrm{H}$-bonded one. After deconvolution of this band, $73 \%$ of the amide groups were estimated to remain as non-H-bonded in these films at $30 \mathrm{mN} / \mathrm{m}$ (Fig. S1C in the Supplementary Material). This remarkable difference in intermolecular H-bonding between 16:0 and 24:1-SM molecules is consistent with calorimetric studies of the hydration of the polar groups of pure SM bilayers [32] where 16:0-SM was found to bind only 5.5 molecules of $\mathrm{H}_{2} \mathrm{O} /$ lipid compared to 22/24-SM that binds 9 molecules of $\mathrm{H}_{2} \mathrm{O}$ /molecule at comparable levels of hydration.

The phosphate group region between 1000 and $1300 \mathrm{~cm}^{-1}$ on panel $\mathrm{C}$ in Fig. 3 was also inspected. In agreement with other studies [30,33] for 16:0-SM, the band at $1230 \mathrm{~cm}^{-1}$ assigned to antisymmetric $\mathrm{PO}_{2}^{-}$ stretching had two components at 1250 and $1219 \mathrm{~cm}^{-1}$ at the two surface pressures studied, corresponding to dehydrated and hydrated $\mathrm{PO}_{2}{ }^{-}$groups, respectively. The symmetric $\mathrm{PO}_{2}{ }^{-}$stretching band appeared at $1080 \mathrm{~cm}^{-1}$ at $10 \mathrm{mN} / \mathrm{m}$ which did not appear at $30 \mathrm{mN} / \mathrm{m}$, while a small band at $1106 \mathrm{~cm}^{-1}$ remained. The diester phosphate stretching band at $1054 \mathrm{~cm}^{-1}$ for $16: 0-\mathrm{SM}$ films in the $L e$ phase increased its intensity and shifted to $1061 \mathrm{~cm}^{-1}$ in the $L c$ phase, which could be a consequence of changes in orientation of the phospholipid headgroup [34]. In contrast, 24:1-SM monolayers at $30 \mathrm{mN} / \mathrm{m}$ showed a broad band encompassing the two $\mathrm{PO}_{2}{ }^{-}$antisymmetric stretching at $\sim 1230 \mathrm{~cm}^{-1}$, reflecting a more heterogeneous level of hydration of the $\mathrm{PO}_{2}{ }^{-}$groups. The two main bands in the $1100-1000 \mathrm{~cm}^{-1}$ region were assigned to $\mathrm{PO}_{2}{ }^{-}$symmetric stretching $\left(1076 \mathrm{~cm}^{-1}\right)$ and $\mathrm{C}-\mathrm{O}-\mathrm{P}-\mathrm{O}-\mathrm{C}$ stretching $\left(1045 \mathrm{~cm}^{-1}\right)$.

In summary, the results in Figs. 2 and 3 can be interpreted as follows. The cis double bond between carbons 15 and 16 in 24:1-SM molecule diminishes intermolecular H-bonding and chain packing density. This explains differences in transition temperature observed for liposomes from gel to liquid-crystalline phases $\left(\mathrm{T}_{\mathrm{m}}\right)$, which are $41{ }^{\circ} \mathrm{C}$ and $23 \pm 3{ }^{\circ} \mathrm{C}$ for 16:0- and 24:1-SM, respectively [35]. $\mathrm{T}_{\mathrm{m}}$ of a molecular species of SM is determined by the balance between intermolecular van der Waals and H-bonding interactions, both of which depend on packing constraints in the hydrocarbon tails of the bilayer 
A

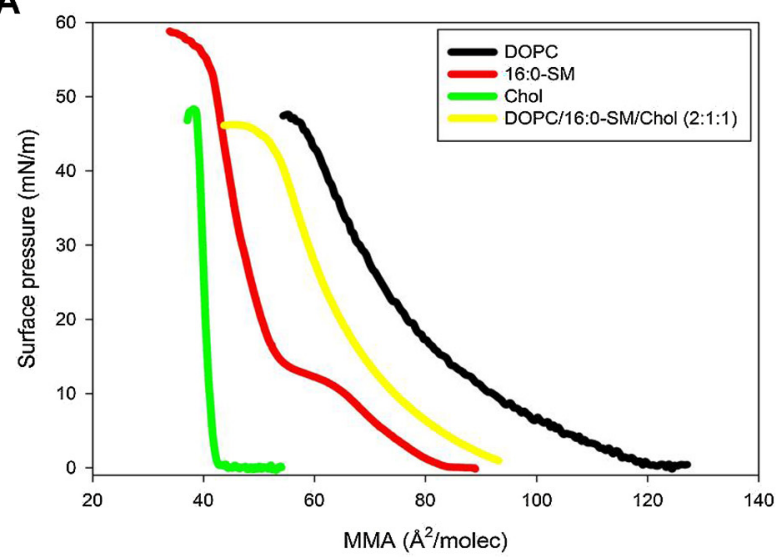

C

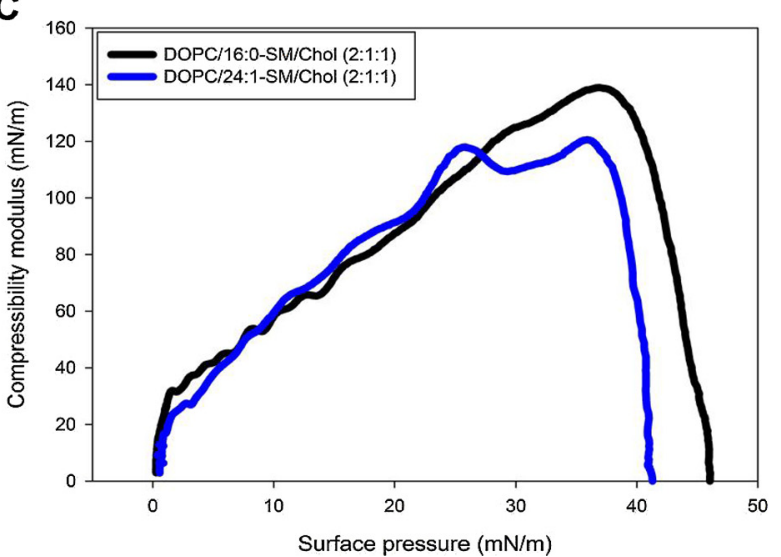

B

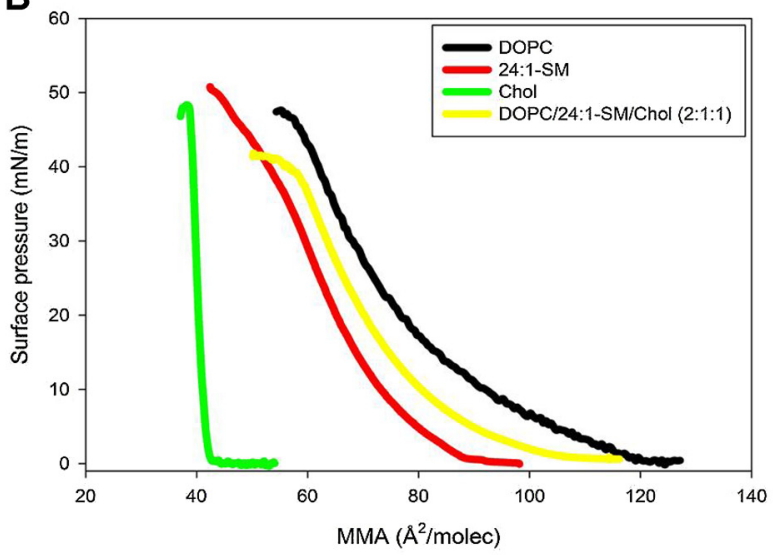

D

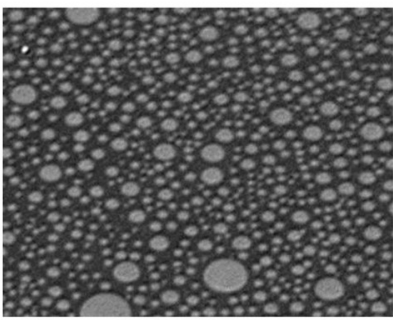

DOPC/16:0-SM/Chol

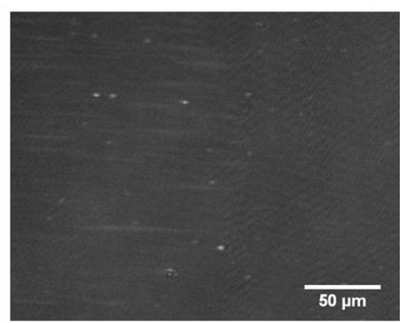

DOPC/24:1-SM/Chol

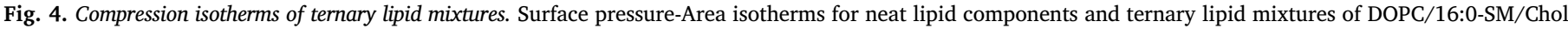

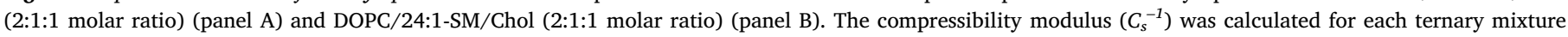

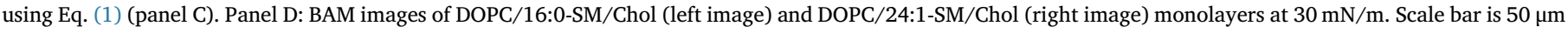
for both images.

[36]. Clearly, these should be key factors that determine the miscibility of SM with PC and Chol.

\subsection{Ternary lipid mixtures}

Monolayers of the canonical raft mixture (PC/SM/Chol) were investigated for the two SMs, whose $\pi$-Area isotherms are shown on panels A and B of Fig. 4. The isotherms for the neat lipid monolayers are consistent with those in the literature [23,24]. The Chol monolayer displayed a very low compressibility with steep increase in $\pi$ while neat DOPC showed a $L e$ behavior. The isotherms of ternary lipid mixtures showed $L e$-like behavior as indicated by the low values of $C_{s}^{-1}$ (Fig. 4, panel C) with MMA values intermediate between those of SM and DOPC. 16:0-SM-containing mixtures collapsed at lower surface pressures $(45.8 \pm 0.4 \mathrm{mN} / \mathrm{m})$ than for neat $16: 0-\mathrm{SM}(62.1 \pm 2.0 \mathrm{mN} / \mathrm{m})$, at values close to the collapse pressure of DOPC $(46.2 \pm 0.9 \mathrm{mN} / \mathrm{m})$. It is worth mentioning that monolayers of DOPC/16:0-SM/Chol exhibit a two-phase ( $L o / L e)$ coexistence, with DOPC being the major component of the Le phase while a SM- and Chol-enriched Lo phase appeared as domains in the ternary mixture $[37,38]$. The Le behavior obtained for DOPC/16:0-SM/Chol films indicated that monolayer compressibility was not highly influenced by the presence of Lo domains; instead, the continuous phase compression properties determined the rheological properties of the two-phase system, a feature that has been reported in other systems where two phases coexist [39]. DOPC/24:1-SM/Chol mixtures formed less stable films, without phase coexistence, which collapsed at lower surface pressures $(41.3 \pm 0.1 \mathrm{mN} / \mathrm{m})$ than the pure lipid films, thus indicating unfavorable interactions between the film components. BAM images of the ternary lipid mixtures at $30 \mathrm{mN} / \mathrm{m}$ confirmed the presence of phase coexistence in DOPC/16:0-SM/Chol films (Fig. 4, panel D, left image) with a thick phase (light gray) forming circular domains immersed in a thinner phase (dark gray). Previous results by Fanani et al. [38] support the assignment of a Lo character to the thick phase and $L e$ character to the thinner continuous phase. On the other hand, the right image in Fig. 4, panel D, shows a completely different microscopic organization and texture of the ternary films containing 24:1-SM, characterized by a homogenous ( $L e$ ) phase.

Considering the phase behavior of both ternary lipid mixtures monolayers at $30 \mathrm{mN} / \mathrm{m}$, their PM-IRRAS spectra shown in Fig. 5 should reflect the presence of both phases $(L e / L o)$ in 16:0-SM-containing monolayers and of a single $L e$ phase in 24:1-SM-containing films. The $\mathrm{CH}$ region for DOPC/16:0-SM/Chol monolayers presented two main bands at 2920 and $2847 \mathrm{~cm}^{-1}$ assigned to $\mathrm{CH}_{2}$ antisymmetric and symmetric stretching vibrations, respectively (Fig. 5, panel A). The $\mathrm{CH}_{2}$ bandwidth for this ternary mixture is larger than for the $\mathrm{CH}$ region of pure 16:0-SM owing to the presence of different $\mathrm{CH}_{2}$ components, as expected for a two lipid-phase system [31]. For DOPC/24:1-SM/Chol films, $\mathrm{CH}_{2}$ bands are observed at 2916 and $2854 \mathrm{~cm}^{-1}$, with $\mathrm{CH}_{3}$ bands appearing as shoulders at $2943\left(\mathrm{CH}_{3}\right.$ antisymmetric $)$ and $2890 \mathrm{~cm}^{-1}$ $\left(\mathrm{CH}_{3}\right.$ symmetric stretching). These results are consistent with 24:1SM-containing monolayers presenting a higher conformational disorder in the acyl chains region, in comparison to 16:0-SM-containing ones. Notwithstanding, the conformational chain order in DOPC/24:1$\mathrm{SM} / \mathrm{Chol}$ films was higher than in pure 24:1-SM monolayers, indicating that incorporation of DOPC and Chol has an ordering effect over 24:1SM. 
A

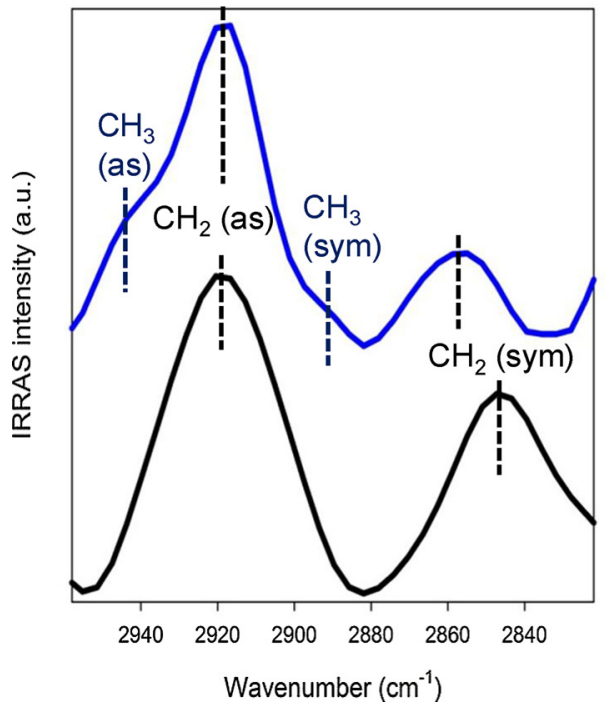

B

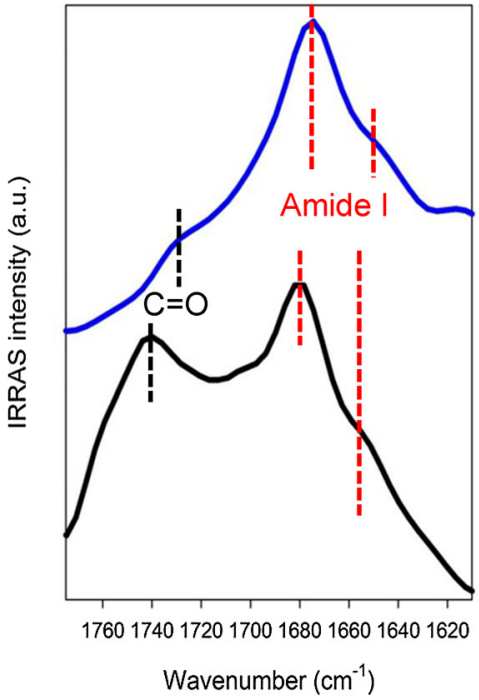

C

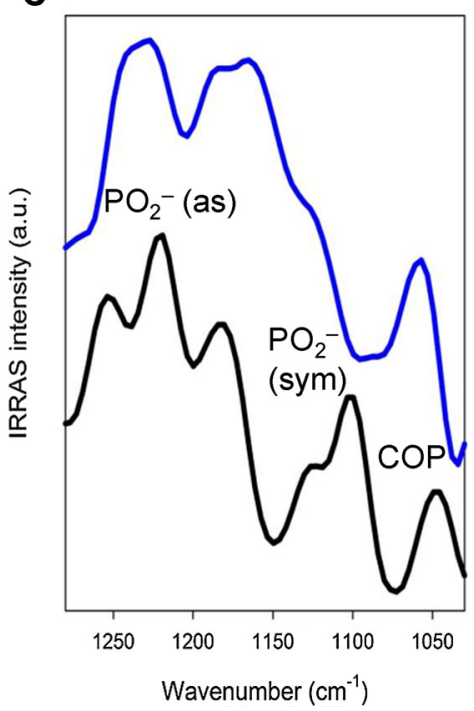

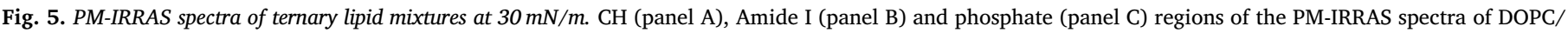

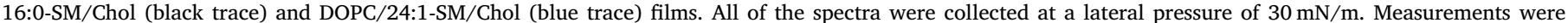
performed at $23 \pm 1{ }^{\circ} \mathrm{C}$ (For interpretation of the references to colour in this figure legend, the reader is referred to the web version of this article).

The $1800-1600 \mathrm{~cm}^{-1}$ region contains information regarding carbonyl and amide I groups, where the carbonyl bands relate to the properties of the phosphatidylcholines at the lipid-water interface (in this case, DOPC) while the Amide I band should depend on the SMwater interface [30]. These bands are strong and occur in a region where Chol itself does not have any major absorption bands. The spectrum for DOPC/16:0-SM/Chol films is markedly different from that of neat 16:0-SM monolayer, featuring an overlap of the two components of the Amide I band at $1682 \mathrm{~cm}^{-1}$ with a shoulder at $1650 \mathrm{~cm}^{-1}$, which could be due to H-bonded amide groups (Fig. 5, panel B). An intense band at $1740 \mathrm{~cm}^{-1}$ is assigned to $\mathrm{C}=\mathrm{O}$ stretching of DOPC. The wavenumbers at which the main bands appeared correspond to non-hydrated DOPC $\mathrm{C}=\mathrm{O}$ groups and mainly non-H-bonded 16:0-SM amide groups. The relative intensity of the low frequency-to-high frequency components of the Amide I band was inverted compared to pure 16:0SM (cf. Fig. 3B). The analysis of the deconvoluted Amide I band indicated that $45 \%$ of the main band corresponded to H-bonded groups in the ternary films (Fig. S2A in the Supplementary Material). In this ternary mixture, 16:0-SM is packed with Chol forming Lo domains. Hence, in addition to SM-SM intermolecular interactions there are also SM-Chol interactions, increasing the heterogeneity of the H-bonding network. This could be the reason for the lower intensity of the $\mathrm{H}$ bonded band, although conformational changes should not be discarded. On the other hand, the Le phase is composed mainly by DOPC whose $\mathrm{C}=\mathrm{O}$ stretching mode had an intensity similar to that of $\mathrm{SM}$ Amide I band. This band was shifted to higher wavenumbers compared to the case of neat DOPC monolayers at $30 \mathrm{mN} / \mathrm{m}$ for which $\mathrm{C}=\mathrm{O}$ vibration was observed at $1732 \mathrm{~cm}^{-1}$ (Fig. S3 in the Supplementary Material), indicating that the addition of SM and Chol affected the behavior of DOPC at the interface which $\mathrm{C}=\mathrm{O}$ groups appeared in a more hydrophobic environment.

In DOPC/24:1-SM/Chol monolayers, an intense Amide I band at $1674 \mathrm{~cm}^{-1}$ with a slight shoulder at $1647 \mathrm{~cm}^{-1}$ was detected. These wavenumbers correspond to non-H-bonded and H-bonded amide groups, respectively. A higher proportion of non-H-bonded groups was confirmed by deconvolution of the amide I band where $77 \%$ of the integrated area corresponded to the non-H-bonded component (Fig. S2B in the Supplementary Material). A strikingly low intensity of the C= $\mathrm{O}$ band of DOPC was observed, detected as a shoulder at $1728 \mathrm{~cm}^{-1}$. This red shift could reflect formation of $\mathrm{H}$-bonds and/or conformational changes that displace these groups to microenvironments of different polarities. In this context, it is worth mentioning that-as stated in the Experimental Section-the intensity of the PM-IRRAS signal is sensitive to the orientation of the vibration moments. Upward bands are observed when the vibration moments lie parallel to the interface. Thus, the low intensity of the $\mathrm{C}=\mathrm{O}$ band in 24:1-SM-containing films indicates that the orientation of DOPC polar groups is different from the one adopted in 16:0-SM-containing monolayers. 24:1-SM is able to accommodate both DOPC and Chol, forming a single phase in monolayers and bilayers [16,24]; therefore, in contrast to what happens in ternary mixtures containing 16:0-SM, in 24:1-SM-containing monolayers no preferential interactions take place between the lipid components. The components are brought together in a single phase, in a process that must involve the adoption of different orientations of the individual lipids compared to the 16:0-SM-containing monolayers.

In the $\mathrm{PO}_{2}{ }^{-}$region, two well-defined bands $\left(1253,1219 \mathrm{~cm}^{-1}\right)$ were observed in DOPC/16:0-SM/Chol ternary monolayers spectrum (Fig. 5, panel C) corresponding to the $\mathrm{PO}_{2}{ }^{-}$antisymmetric stretching, in a similar trend as for pure 16:0-SM monolayers with a minor shift of the high frequency component. A band at $1100 \mathrm{~cm}^{-1}$ with a shoulder at $1119 \mathrm{~cm}^{-1}$ was detected for the symmetric stretching in the ternary mixture with higher intensity than for pure 16:0-SM, while the phosphodiester stretching appeared red-shifted compared to $16: 0$-SM at $1050 \mathrm{~cm}^{-1}$. For DOPC/24:1-SM/Chol monolayers, a band at $1226 \mathrm{~cm}^{-1}$ is assigned to antisymmetric stretching, with the same contour characteristics as in the 24:1-SM monolayer spectra. Thus, the presence of DOPC did not modify the band shape for the phosphate group of 24:1-SM which may indicate that the phosphate group of DOPC could participate in H-bonding with donor groups, most likely the $-\mathrm{OH}$ group of $\mathrm{Chol}$ and/or the $-\mathrm{OH}$ group of SM, since the Amide I band appeared mainly as non-H-bonded groups [33]. This interaction could explain the conformational changes at the interface in this mixture (cf. Fig. 5B). In contrast to pure 24:1-SM monolayers, no clear bands assigned to $\mathrm{PO}_{2}{ }^{-}$symmetric stretching were detected while the band corresponding to phosphodiester stretching mode appeared shifted to higher frequencies, at $1057 \mathrm{~cm}^{-1}$. In both ternary mixtures, the band at $\sim 1165 \mathrm{~cm}^{-1}$ could be attributed mainly to the ester $\mathrm{C}-\mathrm{O}$ asymmetric stretching of DOPC [40].

The results from compression isotherms and PM-IRRAS measurements revealed that the cis double bond in 24:1-SM produced a loosening of lipid chain packing - as evidenced by the higher area per molecule and acyl chain disorder in the ternary lipid films containing 
24:1-SM (cf. Figs. 4 and 5)-with a pronounced effect on the level of hydration at the interface. In the interfacial region, the $\mathrm{C}=\mathrm{O}$ and $\mathrm{PO}_{2}{ }^{-}$stretching vibrations are sensitive to the local environment and the frequencies at which these bands appear are influenced by the level of hydration. For DOPC/24:1-SM/Chol monolayers, the $\mathrm{C}=\mathrm{O}$ stretching band of DOPC at $1728 \mathrm{~cm}^{-1}$ corresponds to fully hydrated carbonyl groups while in DOPC/16:0-SM/Chol films this band appeared at $1740 \mathrm{~cm}^{-1}$ owing to highly dehydrated $\mathrm{C}=\mathrm{O}$ groups at the interface. The analysis of the antisymmetric $\mathrm{PO}_{2}{ }^{-}$bands also revealed a high level of hydration at the interface for 24:1-containing ternary films with the band centered at $\sim 1226 \mathrm{~cm}^{-1}$ appearing due to hydrated $\mathrm{PO}_{2}{ }^{-}$ groups whereas in DOPC/16:0-SM/Chol films a second population of highly dehydrated $\mathrm{PO}_{2}{ }^{-}$groups was detected as a band at $1253 \mathrm{~cm}^{-1}$. Thus, the disordering effect caused by unsaturation in 24:1-SM prevented phase segregation in the ternary mixtures leading to the formation of $L e$ films with high levels of hydration.

Results obtained from PM-IRRAS measurements also revealed a different orientation of DOPC when mixed in ternary mixtures with Chol and the unsaturated asymmetric 24:1-SM than that observed when 16:0-SM is present. The extremely low intensity of the $\mathrm{C}=\mathrm{O}$ band in the 24:1-SM-containing films indicates a reorientation of the $\mathrm{C}=\mathrm{O}$ transition moments with respect to the surface plane. The orientational properties of the $\mathrm{C}=\mathrm{O}$ groups reflect a different conformation of the polar region of DOPC molecules in these ternary mixtures compared to that adopted in DOPC/16:0-SM/Chol and neat DOPC films. In the latter, the intense positive $\mathrm{C}=\mathrm{O}$ stretching bands point to a preferential orientation of $\mathrm{C}=\mathrm{O}$ transition moments on the surface plane. Therefore, the interaction of DOPC with 24:1-SM and Chol in the single Le phase of DOPC/24:1-SM/Chol monolayers seems to force the polar groups of DOPC to adopt a different conformation from that in pure DOPC monolayers or in systems where this PC is the major component of one of the lipid phases - as is the case of DOPC/16:0-SM/Chol mixtures.

\section{Conclusions}

The most important conclusion to infer from the surface pressure isotherms and PM-IRRAS spectra is associated with the importance of acyl chain composition of SM in monolayers with components roughly simulating rafts. Not only were the conformational properties of acyl chains and interfacial hydration affected by 24:1-SM unsaturation but also the orientation of the chemical groups at the air/water interface. This implies that-to obtain a more accurate perspective of membrane lipid phase behavior-it is necessary to examine the properties of different molecular species of lipids from biological sources. In the case of 24:1-SM, the properties conferred by unsaturation have implications for the formation of lipid domains in cell membranes. Unlike 16:0-SM, when 24:1-SM is mixed with DOPC and Chol, a single Le phase is obtained in monolayers with 24:1-SM amide groups being mostly non- $\mathrm{H}-$ bonded. In this regard, due to their H-bonding capability, sphingolipids constitute important molecules involved in cell signaling events [41] and also serve as receptors for various extracellular ligands. SM, for instance, has been found to constitute the binding site for viral proteins [42] and several pore-forming toxins [43-48] with the H-bonding groups (2-NH and the 3-OH) being important for such binding. Results obtained here indicate that the incorporation of 24:1-SM affects the monolayer physical properties and enhances the overall H-bonding ability. The latter may play a key role in cell signaling and binding of biomolecules. We hope that the approach and results presented here may inspire further studies of membrane proteins that depend on the surrounding lipid environment for optimal function.

\section{Acknowledgements}

We thank Mario Raúl Ramos for the graphic designs. This work was supported by the Agencia Nacional de Promoción Científica y Tecnológica [grant number PICT 2657/2013, 1627/2014 and 0679/
2016], the Consejo Nacional de Investigaciones Científicas y Técnicas [PIP 0671], the Comisión de Investigaciones Científicas de la Provincia de Buenos Aires (CICBA), the Universidad Nacional de La Plata [grant number M11/181, M11/191 and 11/X760], the Universidad Nacional de Córdoba (SECyT_UNC), and FAPESP [grant number 2013/14262-7] and CNPq (Brazil). R.V. is a researcher of the Universidad Nacional de La Plata (UNLP), Argentina. M.E.V. is a member of the Carrera del Investigador CICBA, Argentina. M.A.DM., M.L.F. and S.M. are members of the Carrera del Investigador of CONICET.

\section{Appendix A. Supplementary data}

Supplementary material related to this article can be found, in the online version, at doi:https://doi.org/10.1016/j.colsurfb.2018.10.018.

\section{References}

[1] F.M. Goñi, Biochim. Biophys. Acta 1838 (6) (2014) 1467-1476.

[2] K. Simons, G. van Meer, Biochemistry 27 (17) (1988) 6197-6202.

[3] K. Simons, E. Ikonen, Nature 387 (1997) 569-572.

[4] R.E. Brown, J. Cell Sci. 111 (1998) 1-9.

[5] P.J. Quinn, Prog. Lipid Res. 49 (4) (2010) 390-406.

[6] J.V. Busto, J. Sot, J. Requejo-Isidro, F.M. Goñi, A. Alonso, Biophys. J. 99 (2010) 1119-1128.

[7] M.C. Giocondi, D. Yamamoto, E. Lesniewska, P.E. Milhiet, T. Ando, C. Le Grimellec, Biochim. Biophys. Acta 1798 (2010) 703-718.

[8] T. Rog, M. Pasenkiewicz-Gierula, I. Vattulainen, M. Kartunnen, Biochim. Biophys. Acta 1788 (2009) 97-121.

[9] S. Jaikishan, A. Björkbom, J.P. Slotte, Biochim. Biophys. Acta 1798 (2010) 1987-1994.

[10] J. Juhasz, J.H. Davis, F.J. Sharom, Biochem. J 430 (2010) 415-423.

[11] S. Jaikishan, J.P. Slotte, Biochim. Biophys. Acta 1808 (2011) 1940-1945.

[12] J.P. Slotte, Prog. Lipid Res. 52 (2013) 206-219.

[13] J.P.J. Boegheim, M. van Linde, J.A.F. op den Kamp, B. Roelofsen, Biochim. Biophys. Acta 735 (1983) 438-442.

[14] V. Fitzgerald, M.L. Blank, F. Snyder, Lipids 30 (1995) 805-809.

[15] F. Babin, P. Sarda, B. Limasset, B. Descomps, D. Rieu, F. Mendy, A. Crastes de Paulet, Lipids 28 (1993) 627-630.

[16] S. Maté, J.V. Busto, A.B. García-Arribas, J. Sot, R. Vazquez, V. Herlax, C. Wolf, L. Bakás, F.M. Goñi, Biophys. J. 106 (12) (2014) 2606-2616.

[17] R.M. Epand, R.F. Epand, Chem. Phys. Lipids 132 (2004) 37-46.

[18] P.S. Niemelä, M.T. Hyvönen, I. Vattulainen, Biochim. Biophys. Acta 1788 (1) (2009) $122-135$.

[19] J.P. Slotte, Biochim. Biophys. Acta 1858 (2) (2016) 304-310.

[20] G.L. Gaines, Insoluble Monolayers at Liquid-Gas Interfaces, Interscience Publishers, 1966.

[21] C. Lheveder, J. Meunier, H. Henon, W.N.E.A. Baszkin, Brewster Angle Microscopy, Physical Chemistry of Biological Interfaces, Marcel Dekker Inc., NY, 2000.

[22] F.J. Pavinatto, C.P. Pacholatti, E.A. Montanha, L. Caseli, H.S. Silva, P.B. Miranda, T. Viitala, O.N. Oliveira, Langmuir 25 (17) (2009) 10051-10061.

[23] X.M. Li, J.M. Smaby, M.M. Momsen, H.L. Brockman, R.E. Brown, Biophys. J. 78 (2000) 1921-1931.

[24] S.M. Maté, R.F. Vazquez, S.V. Herlax, M.L. Fanani, M.E. Vela, B. Maggio, L. Bakás, Biochim. Biophys. Acta 1838 (2014) 1832-1841 D.-M. M.A..

[25] D. Marsh, Biochim. Biophys. Acta 1286 (1996) 183-223.

[26] R.G. Snyder, A.L. Aljibury, H.L. Strauss, J. Chem. Phys. 81 (12) (1984) 5352-5361.

[27] R. Mendelsohn, J.W. Brauner, A. Gericke, Annu. Rev. Phys. Chem. 46 (1995) $305-334$.

[28] C.L. Leverette, R.A. Dluhy, Colloids Surf. A 243 (2004) 157-167.

[29] M. Rujoi, D. Borchman, D.B. DuPré, M.C. Yappert, Biophys. J. 82 (6) (2002) 3096-3104.

[30] Z. Arsov, L. Quaroni, Biochim. Biophys. Acta 1778 (4) (2008) 880-889.

[31] B. Boulgaropoulos, Z. Arsov, P. Laggner, G. Pabst, Biophys. J. 100 (9) (2011) 2160-2168.

[32] M. Kodama, M. Abe, Y. Kowasaki, S. Ohira, H. Nozaki, C. Katagiri, Thermochim. Acta 416 (2004) 105-111.

[33] J. Villalaín, A. Ortiz, J.C. Gómez-Fernández, Biochim. Biophys. Acta 941 (1) (1988) 55-62.

[34] F.M. Goñi, J.L. Arrondo, Faraday Discuss. Chem. Soc. 81 (1986) 117-126.

[35] N. Jiménez-Rojo, A.B. García-Arribas, J. Sot, A. Alonso, F.M. Goñi, Biochim. Biophys. Acta 1838 (2014) 456-464.

[36] P.J. Quinn, C. Wolf, Biochim. Biophys. Acta 1788 (9) (2009) 1877-1889.

[37] R.F. de Almeida, A. Fedorov, M. Prieto, Biophys. J. 85 (4) (2003) 2406-2416.

[38] M.L. Fanani, B. Maggio, J. Phys. Chem. 115 (2011) 41-49.

[39] N. Wilke, F. Vega Mercado, B. Maggio, Langmuir 26 (13) (2010) 11050-11059.

[40] J.E. Fookson, D.F. Wallach, Arch. Biochem. Biophys. 189 (1) (1978) 195-204.

[41] N. Bartke, Y.A. Hannun, J. Lipid Res. 50 (Suppl) (2009) 91-96.

[42] L. Weng, Y. Hirata, M. Arai, M. Kohara, T. Wakita, K. Watashi, K. Shimotohno, Y. He, J. Zhong, T. Toyoda, J. Virol. 84 (22) (2010) 11761-11770.

[43] A.W. Bernheimer, L.S. Avigad, Proc. Natl. Acad. Sci. U. S. A. 73 (2) (1976) 467-471. 
[44] T. Tomita, K. Noguchi, H. Mimuro, F. Ukaji, K. Ito, N. Sugawara-Tomita, Y. Hashimoto, J. Biol. Chem. 279 (26) (2004) 26975-26982.

[45] H. Shogomori, T. Kobayashi, Biochim. Biophys. Acta 1780 (3) (2008) 612-618.

[46] L. Garcia-Ortega, J. Alegre-Cebollada, S. Garcia-Linares, M. Bruix, A. Martinez-DelPozo, J.G. Gavilanes, Biochim. Biophys. Acta 1808 (9) (2011) 2275-2288.
[47] T. Takara, T. Nakagawa, M. Isobe, N. Okino, S. Ichinose, A. Omori, M. Ito, Biochim. Biophys. Acta 1811 (5) (2011) 323-332.

[48] L. Pedrera, M.L. Fanani, U. Ros, M.E. Lanio, B. Maggio, C. Alvarez, Biochim. Biophys. Acta 1838 (7) (2014) 1752-1759. 